\title{
Blind Navigation
}

\author{
Adwait Sanjeev \\ Department of Computer \\ Engineering \\ St. John College of Engineering \\ and Management \\ Palghar, India
}

\author{
Mansi Suhas Raut \\ Department of Computer \\ Engineering \\ St. John College of Engineering \\ and Management \\ Palghar, India \\ Angelin Florence A \\ Department of Computer \\ Engineering \\ St. John College of Engineering \\ and Management \\ Palghar, India
}
Arindam Alok Samanta
Department of Computer
Engineering
St. John College of Engineering
and Management
Palghar, India

\begin{abstract}
This paper presents a system for those people of our society who are suffering from visually challenging situations. The system is able to understand obstacles around the subject in the range $2 \mathrm{~cm}-400 \mathrm{~cm}$ using ultrasonic sensors. Sensor are placed on the stick. It calculates distance of the detected obstacle from the user and gives feedback in the form of voice output. The proposed system uses Arduino Uno based system to process real time data collected using ultrasonic sensor network. Based on the detected signal from the obstacle, appropriate pre-recorded voice output is given to the user via Bluetooth module to their smart phone. The feedback is given to the user via earphones.

Keywords: Arduino, Blind people, Bluetooth Module, Ultrasonic Sensors, Earphones.
\end{abstract}

\section{INTRODUCTION}

According to a survey approximately vision impairment is found in 1.3 billion people [1]. Due to ageing population and many changes in lifestyle increased number of people are suffering from blinding conditions such as diabetic retinopathy is projected to rise. Without effective, major intervention, the number of blind people worldwide has been projected to increase to 76 million by 2020 if current trends continue [2]. In present time many traditional navigations systems are used. Usage of all these travel aids for detecting obstacles for smooth navigation requires a good training. Presently several electronic travel aids (ETA) are available for visually impaired and blind people. These aids are designed using recent technological developments in automation.

Although many advanced electronic systems for the visually challenged people are present in the market, only few of them are useful. Therefore, user acceptability assessment of such systems is very important. The important parameters which should be considered in this are size, portability, reliability, useful functionalities, simple user interface, training time, system robustness and affordability in terms of cost. To overcome all these limitations, a system considering all the needs of the user has been proposed in this paper.

\subsection{Motivation}

We often see a blind person having difficult time walking down a busy street. Blind people always needed someone to guide them. They have to be dependent on someone or the other. People are very busy with their own life and they are hardly bothered about anyone else. This makes the life of such blind people very difficult. To help them in such a way that they won't be a burden to anyone again is the need of the hour. So, in the proposed system depicts the idea of smart stick for the blinds which will help them find the way on their own without the help of anyone.

\subsection{Problem Statement}

Helping the blind in navigation outdoors as well as indoors is an important issue. Being self-dependent is the most important trait in an individual in the modern world. The existing systems help the visually impaired people but they are not effective enough. These systems could not detect the obstacles they would encounter while moving forward. They are mostly for the obstacles just lying around. The proposed system will thus aim to solve all these issues and help to make their lives easy and simple.

\subsection{Objectives}

- To design a system to detect obstacles the user would encounter.

- To make the visually impaired people selfdependent.

- To make the life of visually impaired people simple and easy.

- To implement the system as cost efficient

\section{REVIEW OF LITERATURE}

Literature Review acts as the basis of research and study of the various concepts required for a particular domain. It describes the theories and other methodologies that can be 
adopted in order to implement modules of the proposed system.

\subsection{D Ultrasonic Stick for Blind}

Today technology is improving daily in different aspects in order to provide flexible and safe movement for the people. In this technology driven world, where people strive to live independently, this system propose a low-cost 3D ultrasonic stick for blind people to gain personal independence, so that they can move from one place to another easily and safety. A portable stick is design and developed that detects the obstacles in the path of the blind using ultrasonic sensors. It consists of these sensors to scan three different directions, a microcontroller, buzzer and DC vibration motor. The buzzer and vibration motor are activated when any obstacle is detected. In addition, the stick is equipped with GPS and SMS message system. GPS system provide the information regarding the location of the blind person using the stick to his family members. SMS system is used by the blind to send SMS message to the saved numbers in the microcontroller in case of emergency. The programming of GPS modem, GSM modem, buzzer and vibration motor has been successfully done for this system. Computer simulation is done to essence the performance of the system using Proteous software and Easy pic kit.

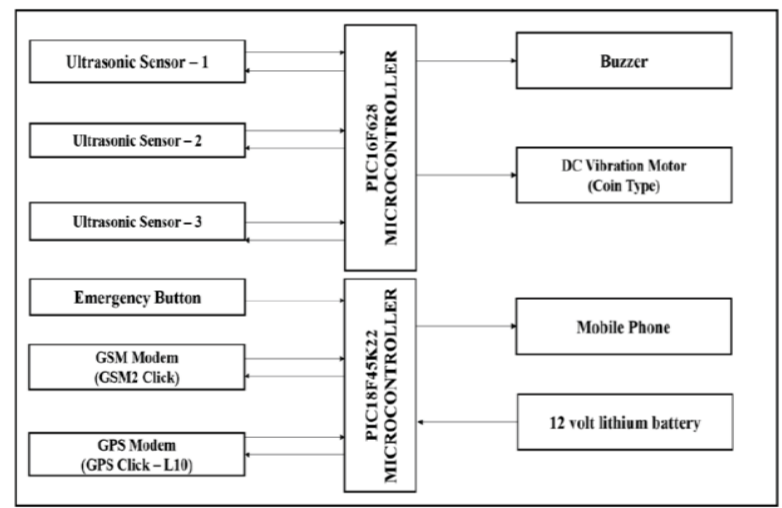

Figure 2.1: Block Diagram for 3D Ultrasonic Stick for Blind

\subsection{Ultrasonic Spectacles and Waist-belt for Visually Impaired and Blind Person}

This system presents an electronic navigation system for visually impaired and blind people (subject). This system understands obstacles around the subject up to $500 \mathrm{~cm}$ in front, left and right direction using a network of ultrasonic sensors. It effectively calculates distance of the detected object from the subject and prepares navigation path accordingly avoiding obstacles. It uses speech feedback to aware the subject about the detected obstacle and its distance. This proposed system uses AT89S52 microcontroller based embedded system to process real time data collected using ultrasonic sensor network. Based on direction and distance of detected obstacle, relevant pre-recorded speech message stored.

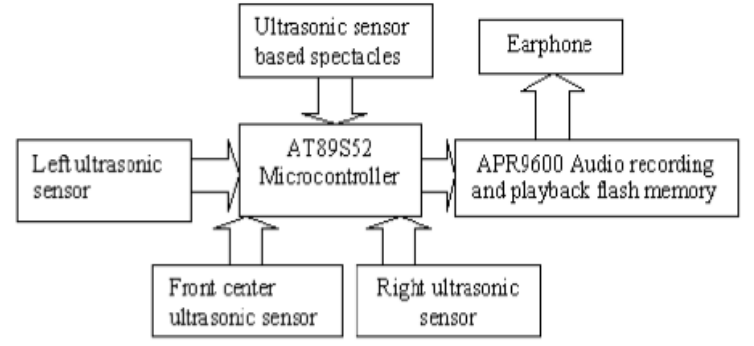

Figure 2.2: Ultrasonic Spectacles and Waist-belt for Visually Impaired and Blind Person

\subsection{Distance Sensing with Ultrasonic Sensor and Arduino}

A sensor is a device that converts one type of energy to another. Arduino is a small microcontroller board with a USB plug to connect to the computer. The Arduino board senses the environment by receiving input from a variety of sensors and can affect its surroundings by controlling LCDs, speakers, motors and GS module. Ultrasonic Sensor measure the distance of target objects or materials through the air using "non-contact" technology. They measure distance without damage and are easy to use. The output Signals received by the sensor are in the analog form, and output is digitally formatted and processed by microcontroller. In present work, it is used to detecting an obstacle, along with its exact distance. The internal analog to digital converter is used is calibrated to get almost accurate distance measurement. The measured distance is also displayed on an LCD screen.

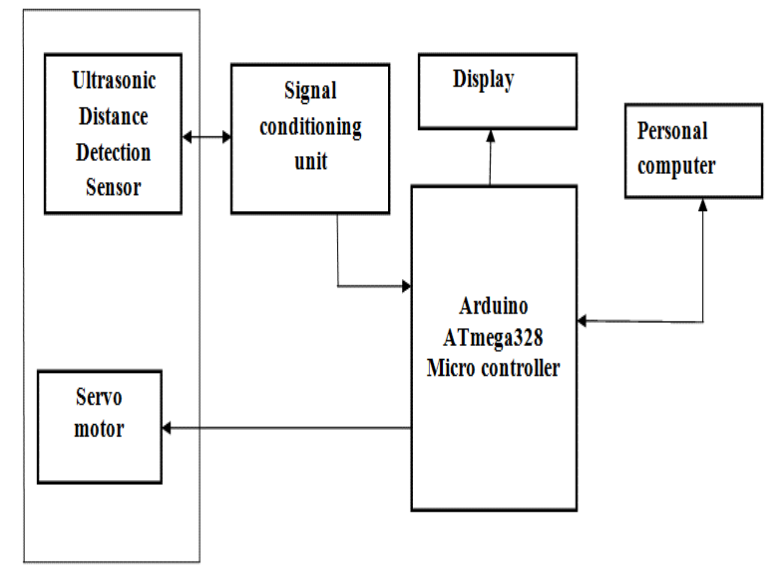

Figure 2.3: Block diagram of Ultrasonic Distance Detection with Arduino

\section{METHODOLGY}

The proposed system concentrates on helping the visually impaired people in navigation in their day to day life and to make their life easy and relatively simple. The proposed system aims to detect objects in the path of the visually 
impaired people and notify them via voice output. This method of object sensing is carried out using ultrasonic sensors. The system will use Ultrasonic sensors interfaced with Arduino Uno R3 [3]. Using Arduino, the detected ultrasonic signals will be converted to numeric value in the form of distance to the detected object. According to the processed signals from the Arduino, the voice messages will be retrieved and voice output through the user's smartphone will be provided to the user via ear phones. The system will thus help them to navigate around surroundings with ease without depending on any other people for help.

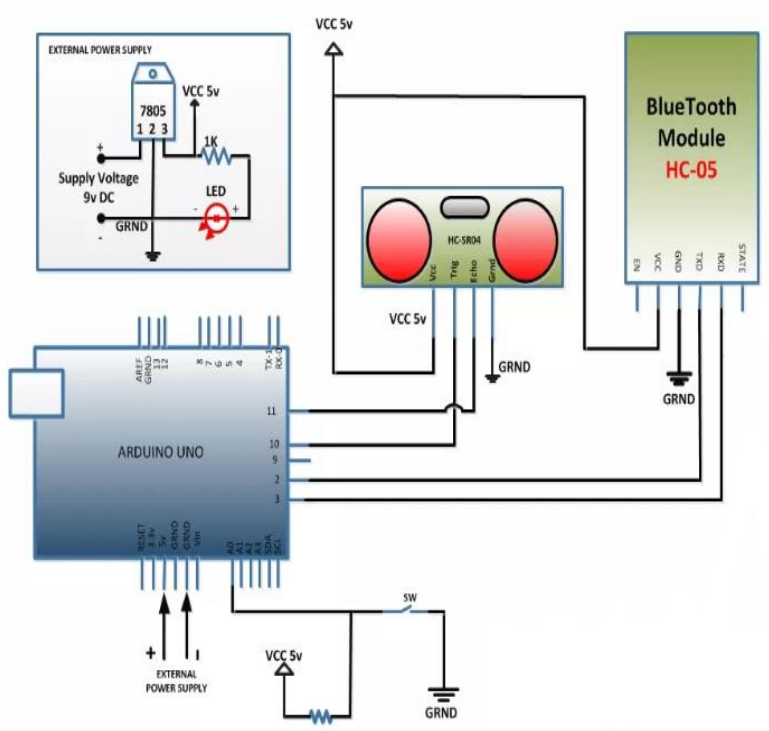

Fig: 3.1 Architecture Diagram

The architecture of the proposed system is as shown in Fig 3.1. The Arduino Uno is the main processing unit of the system. It receives signals from the HC SR-04 sensors. The voice output is transferred to the user's smartphone through the Bluetooth module. The voice output to the user is given through the earphones.

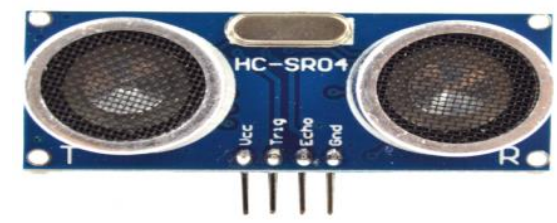

Fig .3.2: HC-SR04 Ultrasonic Sensors

The HC-SR04 ultrasonic sensor uses sonar to determine distance to an object like bats do. It offers excellent noncontact range detection with high accuracy and stable readings in an easy-to-use package.

From $2 \mathrm{~cm}$ to $400 \mathrm{~cm}$ or 1 " to 13 feet. Its operation is not affected by sunlight or black material like sharp rangefinders are (although acoustically soft materials like cloth can be difficult to detect). It comes complete with ultrasonic transmitter and receiver module. The ultrasonic sensors are as shown in Fig 3.2.

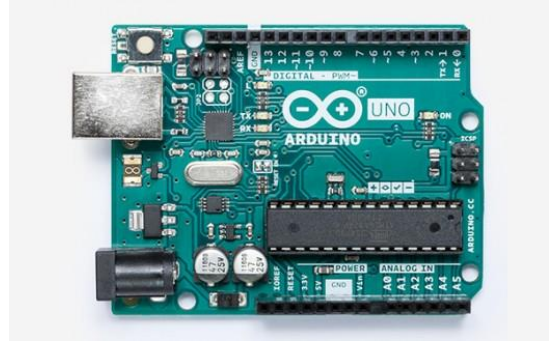

Fig 3.3: Arduino Uno

The Arduino UNO is an open-source microcontroller board based on the Microchip ATmega328Pmicrocontroller and developed by Arduino.cc. The board is equipped with sets of digital and analog input/output (I/O) pins that may be interfaced to various expansion boards (shields) and other circuits. The circuit of Arduino Uno is as shown in Fig: 3.3.

\section{OBSTACLE DETECTION AND COMPONENTS USED}

To help detect the objects in front of the user ultrasonic sensors are used. Ultrasonic sensors work in pairs, one of which is used to transmit ultrasonic signals while the other is used to receive the signals. The working of these sensors is similar to the SONAR system used in ships. Once detected the signals are transmitted to the Arduino Uno. Two ultrasonic sensors are used in this system

\subsection{Software Unit}

\subsubsection{Arduino IDE}

The coding of the entire process is done in this software. It helps the Arduino to carry out the tasks as per the given instructions in the code. It also converts the ultrasonic signals to numeric values in terms of distance from the detected object. Once the code is written it is uploaded in the Arduino IC.

\subsection{Hardware Unit}

\subsubsection{Ultrasonic Sensors (HC SR-04):}

These sensors work in the range of $2 \mathrm{~cm}-400 \mathrm{~cm}$. It consists of two parts one transceiver and one receiver. The transceiver transmits the ultrasonic signals while the receiver receives them. The signals are then given to the Arduino for further processing.

\subsubsection{Arduino UNO}

It will be connected to computer with a USB cable. Thus, the detected signal will be transferred to the Arduino Uno. It also interfaced the APR33A3 voice module. The Arduino converts the signals into numeric values in the form of distance from the detected object.

\subsubsection{Bluetooth Module}

The text string containing the distance of the user from the object is transferred to the Bluetooth module from the Arduino Uno which is then transferred to the user's smartphone and voice output is given to the user.

\subsubsection{Earphones}

Earphones are used to transmit the voice message from the Voice Module to the user. 


\section{WORKING OF THE SYSTEM}

\subsection{Setting Up the System}

Arduino is interfaced with Bluetooth module along with the ultrasonic sensors. The code is uploaded into the Arduino before starting the system. Text messages are transferred to the user's smartphone which are then played as voice output.

\subsection{Working of The System}

Switch on the ultrasonic sensors to start sensing the objects. The ultrasonic sensors will sense the objects in the user's proximity and send the necessary signals to the Arduino. The Arduino processes these signals and converts it to numeric value in the form of distance from the detected object. The distance in the form of text strings are then transferred to the user's smartphone via the Bluetooth module. This message is then played as voice output to the user via earphones.

\section{CONCLUSION}

Using the above methodology, distance of the objects was calculated using Ultrasonic sensor (HC SR-04). The readings were noted based on the distance calculated by the sensors. from the PING) 1): a HIGH

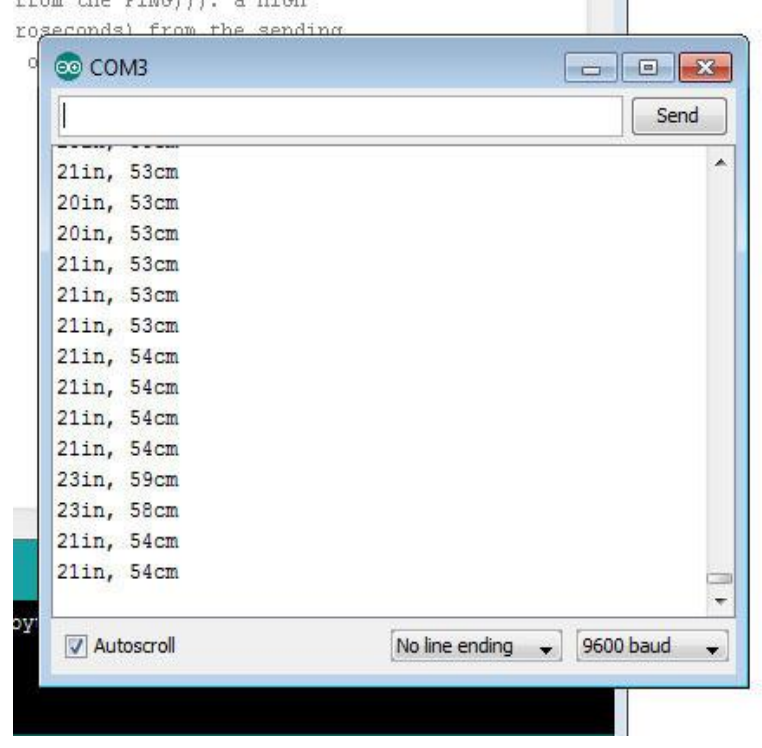

Figure 6.1: Readings as shown on Arduino IDE
Readings were shown in Arduino IDE based on the distance of the object from the sensors. The readings are as shown in Fig 6.1.

\section{REFERENCES}

[1] Blindness and Visual Impairment: Global Facts. https://www.who.int/news-room/factsheets/detail/blindness-and-visual-impairment

[2] Blindness and Visual Impairment: Global Facts. http://www.vision202 -0.org.

[3] N. Anju Latha, B. Rama Murthy, K. Bharat Kumar, "Distance Sensing with Ultrasonic Sensor and Arduino International Journal of Advance Research, Ideas and Innovations in Technology, Volume2, Issue 5, 2016. 